\title{
Structural Basis of Bloom Syndrome (BS) Causing Mutations in the BLM Helicase Domain
}

\author{
Suo-Bao Rong, Jouni Väliaho, and Mauno Vihinen \\ Institute of Medical Technology, University of Tampere, Tampere, Finland and \\ Tampere University Hospital, Tampere, Finland \\ Accepted November 25, 1999.
}

\begin{abstract}
Background: Bloom syndrome (BS) is characterized by mutations within the BLM gene. The Bloom syndrome protein (BLM) has similarity to the RecQ subfamily of DNA helicases, which contain seven conserved helicase domains and share significant sequence and structural similarity with the Rep and PcrA DNA helicases. We modeled the three-dimensional structure of the BLM helicase domain to analyze the structural basis of BS-causing mutations.

Materials and Methods: The sequence alignment was performed for RecQ DNA helicases and Rep and PcrA helicases. The crystal structure of PcrA helicase (PDB entry 3PJR) was used as the template for modeling the BLM helicase domain. The model was used to infer the function of BLM and to analyze the effect of the mutations.

Results: The structural model with good stereo-
\end{abstract}

chemistry of the BLM helicase domain contains two subdomains, $1 \mathrm{~A}$ and $2 \mathrm{~A}$. The electrostatic potential of the model is highly negative over most of the surface, except for the cleft between subdomains $1 \mathrm{~A}$ and $2 \mathrm{~A}$ which is similar to the template protein. The ATP-binding site is located inside the model between subdomains $1 \mathrm{~A}$ and 2A; whereas, the DNA-binding region is situated at the surface cleft, with positive potential between 1A and $2 \mathrm{~A}$.

Conclusions: The three-dimensional structure of the BLM helicase domain was modeled and applied to interpret BS-causing mutations. The mutation I841T is likely to weaken DNA binding, while the mutations C891R, C901Y, and Q672R presumably disturb the ATP binding. In addition, other critical positions are discussed.

\section{Introduction}

Bloom syndrome (BS, MIM\# 210900) is a rare human recessive disorder associated with growth retardation, immunodeficiency and increased risk of malignancy at an early age $(1,2)$. The causative gene for $\mathrm{BS}$ is $B L M$, which was mapped to the long arm of chromosome 15 (15q26.1), and encodes a protein with 1417 amino acids (3). The Bloom syndrome (BLM) protein consists of seven domains (Fig. 1), from the amino terminus poly-aspartate domain (PD 1), poly-serine domain (PS), poly-aspartate domain (PD2), DEAH helicase domain (DEAH) (4), RecQ helicase C-terminal domain

Address correspondence and reprint requests to: Prof. Mauno Vihinen, Institute of Medical Technology, University of Tampere, FIN-33104 Tampere, Finland. Phone: 358-3-2157735; Fax: 358-3-2157710; E-mail: mauno.vihinen@uta.fi
(RecQCt) (4), helicase and RNase D C-terminal domain (HRDC) (5), and nuclear localization signals (NLS) (6). The BLM protein shows similarity to sequences of the RecQ subfamily of ATP-dependent DNA helicases, including RecQ (7), Sgsl $(8,9)$, Rqhl (10), and the human RECQL $(11,12)$ and WRN (13) proteins.

DNA helicases catalyze the unwinding of double-stranded DNA to provide singlestranded templates for replication, repair, recombination and transcription $(14,15)$. Most helicases contain seven conserved helicase motifs. The first and third motifs, referred to as I and II, coexist in proteins that bind nucleotide triphosphates and they are not specific to helicases (16). The other motifs, Ia and III-VI, are unique to helicases, presumably reflecting conserved protein structures that mediate DNA binding and unwinding. The BLM protein exhibits an ATP-dependent DNA helicase activity 


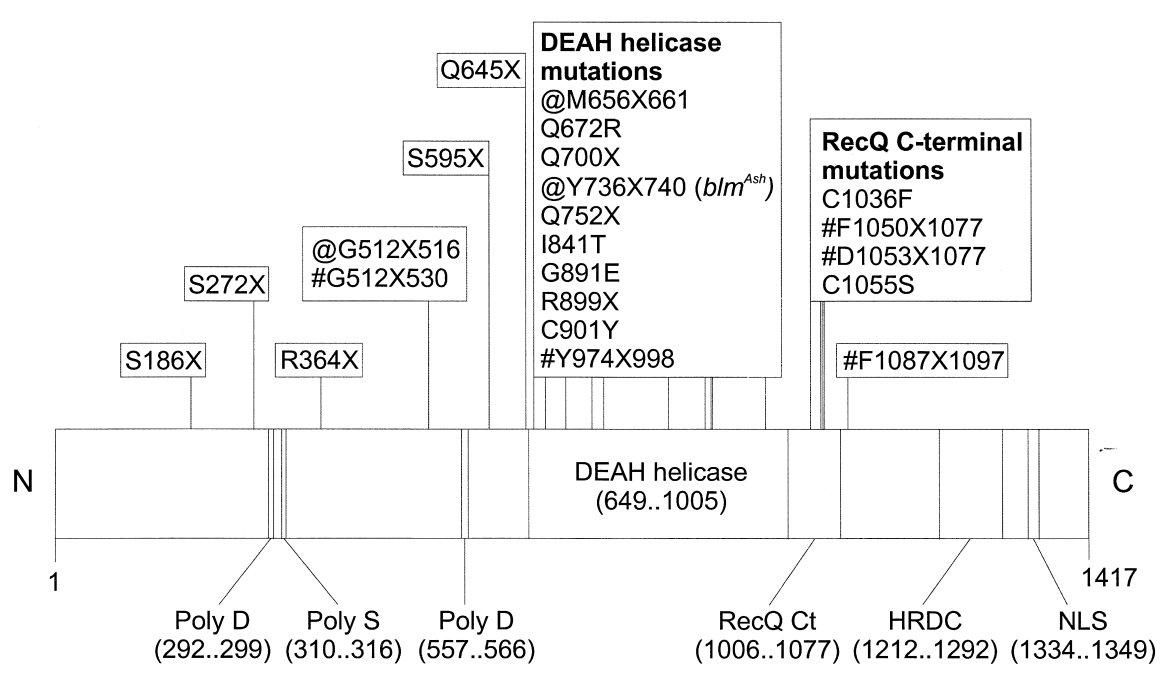

Fig. 1. Schematic depiction of distribution of the wild-type BLM protein and identified mutant alleles. All currently known mutations are presented in boxes according to convention used in Vihinen et al. (50). Insertions are marked with the "@" sign and deletions with "\#" in front of the mu-

tation site. If the mutation causes a stop codon, it is marked with $X$ and the number of the codon with the newly introduced stop codon. The mutation positions are indicated by vertical lines. Domain organization of BLM protein is shown below mutations.

that unwinds DNA in a $3^{\prime}-5^{\prime}$ direction (17) like WRN $(18)$ and Sgsl proteins $(19,20)$.

BLM mutations lead to excessive chromosome breakage and increased rates of sister chromatid interchange in somatic cells (2). Although many BS mutations truncate the BLM protein upstream of the helicase domain, four single amino acid substitutions in helicase domain, namely Q672R, I841T, G891E, and C901Y, have been reported on the human BLM $(3,21,22)$ and three point mutations, K703A, Q680P, and I849T, on the mouse BLM (4). These substitutions abolish both ATPase and DNA unwinding activities of BLM protein.

Recently, the crystal structures were determined for Escherichia coli Rep (23) and Bacillus stearothermophilus PcrA DNA helicases (24-26). These DNA helicases share significant sequence and structural similarity and contain all the signature helicase motifs $(23,24,27,28)$. The crystal structures, including complexes with single strand DNA (ssDNA), provide insights into the interactions of helicases with DNA. The ssDNA binding involves residues from the helicase motifs Ia, III and V; whereas, the ATP binding involves the helicase motifs I and IV. Additionally, mutations in a number of conserved helicase motifs around the ATPase active site were characterized for Rep and PcrA DNA helicases and revealed possible roles for amino acid residues in the active site $(23,25,26)$.

The recent crystal structures of DNA helicases, as well as the molecular biological knowledge of BLM, were combined to shed light on the effect of the BS-causing mutations in the BLM helicase domain. We modeled the helicase domain structure of the BLM protein on the basis of the crystal structures of DNA helicases and studied the structural basis of BS-causing mutations.

\section{Materials and Methods}

\section{BLM Mutation Database}

A program was developed for the submission of mutation and patient information to BLMbase. The registry was built according to the guidelines adopted in BTKbase (IMT, University of Tampere, Finland) (29). The aim was to provide information about the BS patients and their genetic defects on the Internet by using the World Wide Web. The BLMbase contained four main items: identification of the patient and mutation(s), reference either to published article(s) or a submitting physician, mutation information, and data related to disease and therapy. All the items were organized as fields, which were easily accessible. Protection of the patient's identity was assured in the registry. Patient entries could be analyzed with the provided tools or with a sequence retrieval system (SRS) (30). 


\section{Modeling of BLM Helicase Structure}

The sequences of RecQ subfamily DNA helicases WRN (SWISS_PROT: Q14191), BLM (P54132), RECQL (P46063), SGS1 (P35187), RecQ (P15043) and Rqhl(Q09811) were retrieved from sequence databases. Their sequence comparison information $(3,4,10,15)$ was used to perform sequence alignment with Rep and PcrA DNA helicases. The crystal structures of Rep (PDB entry IUAA) and PcrA DNA helicases (1PJR, 2PJR, 3PJR, 1QHG and $1 \mathrm{QHH}$ ) were structurally conserved on seven helicase domains with high sequence similarity.

The multiple sequence alignment was performed for the RecQ subfamily, Rep and PcrA helicases using Clustal W (31). The final alignment was achieved through manual adjustment, based on the conservation of the helicase motifs. The PcrA DNA helicase (3PJR) was used as the template for modeling, because of the information concerning ATP and DNA binding (26).

The interactive modeling and display were performed on Silicon Graphics OCTANE with InsightII program (Molecular Simulations, Inc., San Diego, CA). The conserved regions of the BLM protein were modeled based on the template protein. Sidechain rotamer library was used to model amino acid substitutions. Deletions and insertions were built up by searching the loop database derived from Protein Data Bank (PDB) files $(32,33)$. The program Discover (Molecular Simulations, Inc.) was employed for model refinement. The initial model was optimized stepwise, with energy minimization using an Amber force field (University of California, San Francisco, CA). First, the loops with fixed and freed backbone were respectively minimized for $\mathbf{5 0 0}$ steps. Afterwards, 500 steps of minimization were performed on the whole model with constrained backbone, and then 800 steps with fixed $C_{\alpha}$ atoms. The final model was checked for its quality using the program PROCHECK (University College, London, UK) (34).

\section{Results}

\section{BLM Mutation Database}

All the reported BS-causing mutations (3,21,22, 35-38) were collected into a database called BLMbase. Currently, 32 mutation entries from 31 unrelated families showing 22 unique genotypes are listed. In addition to mutations, the registry contains information also about symptoms, age at diagnosis and various relevant parameters from the patient. The registry is constructed according to the concepts used in BTKbase, X-linked agammaglobulinemia (XLA) mutation registry (29) by using the MUTbase system (IMT, University of Tampere, Finland) (39). The BLMbase is freely accessible on the World Wide Web at http:// www.uta.fi/imt/bioinfo/BLMbase/. Researchers are encouraged to send their patient and mutation information to the registry. BLMbase provides additional information, such as alterations in restriction enzyme patterns due to mutations and mutation statistics. The reported mutations are scattered into several domains.

\section{Sequence Alignment}

To understand the structural consequences of BS, the structure of the BLM helicase domain was modeled. The sequence alignment among the seven helicase domains of the RecQ DNA helicases and the candidate templates was based on the conservation of helicase motifs and was adjusted according to the secondary structure. The secondary structure of the BLM protein was predicted at Internet server Jpred $(40,41)$. The secondary structure of Rep and PcrA DNA helicases was taken from PDBsum database (Research Collaboratory Structural Bioinformatics) (42) and is shown in Figure 2. The sequence alignment (Fig. 2) shows that the seven conserved helicase domains sequentially match each other. Furthermore, the distribution of the predicted secondary structural elements of the BLM protein was similar to that of the Rep and PcrA DNA helicases.

In the sequence alignment, there were three insertions of 4,8 , and 32 residues and six deletions of 7, 3, 77, 11, 215, and 6 residues, all of which were located in the variable loops on the surface of protein connecting neighboring secondary structures or subdomains. The insertion of 32 residues was not modeled due to its size. The deletion of 77 residues corresponded to the surface-located subdomain 1B, between residues 108 and 188 in PcrA helicase; whereas, the deletion of 215 residues included a quarter of the surface-exposed subdomain $2 \mathrm{~A}$, from residues 324 to 384 and the whole subdomain $2 \mathrm{~B}$, extending from residues 385 to 559 .

\section{Structural Model}

The helicase domain of the BLM protein was modeled on the basis of the PcrA core struc- 

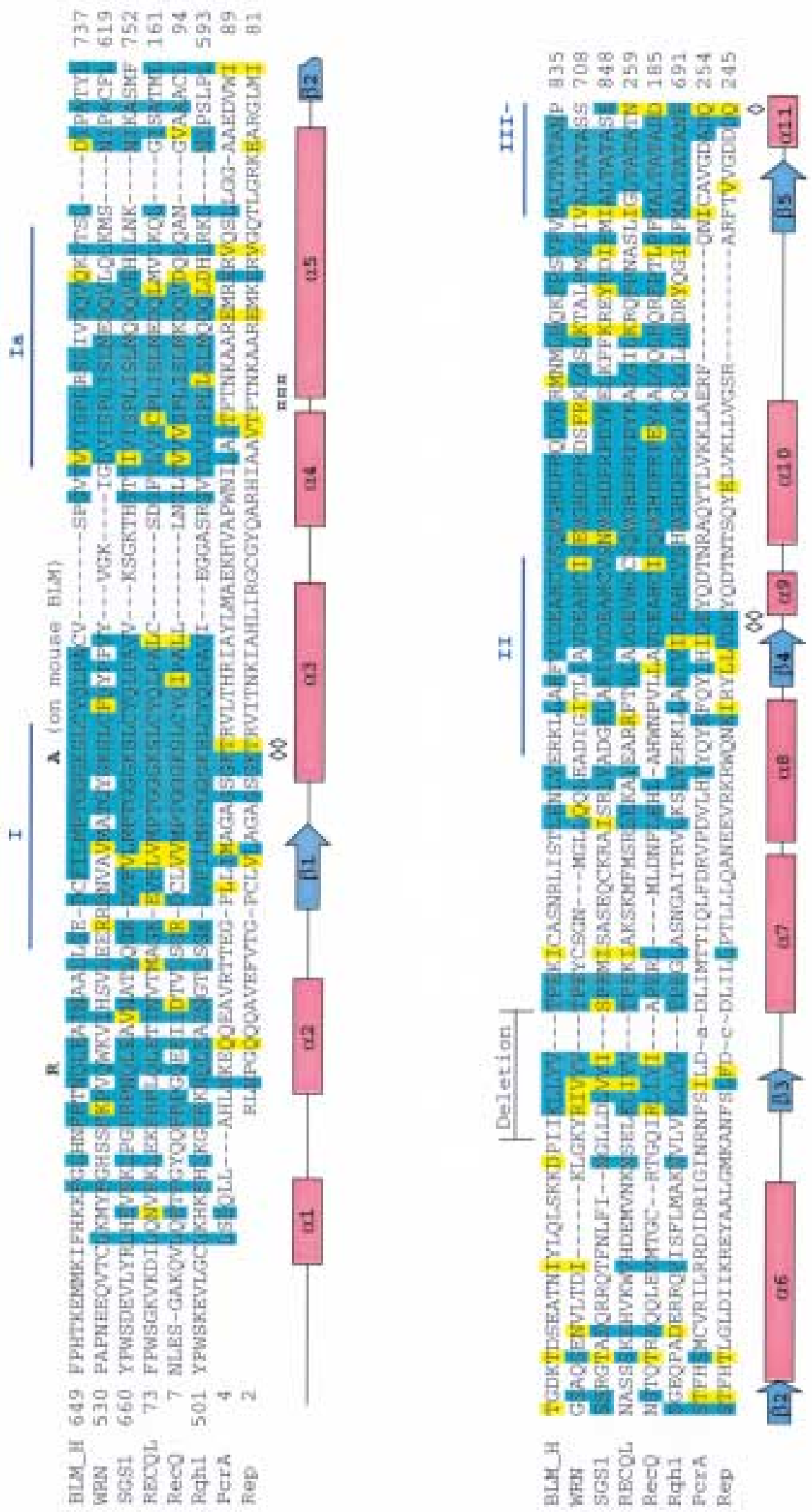

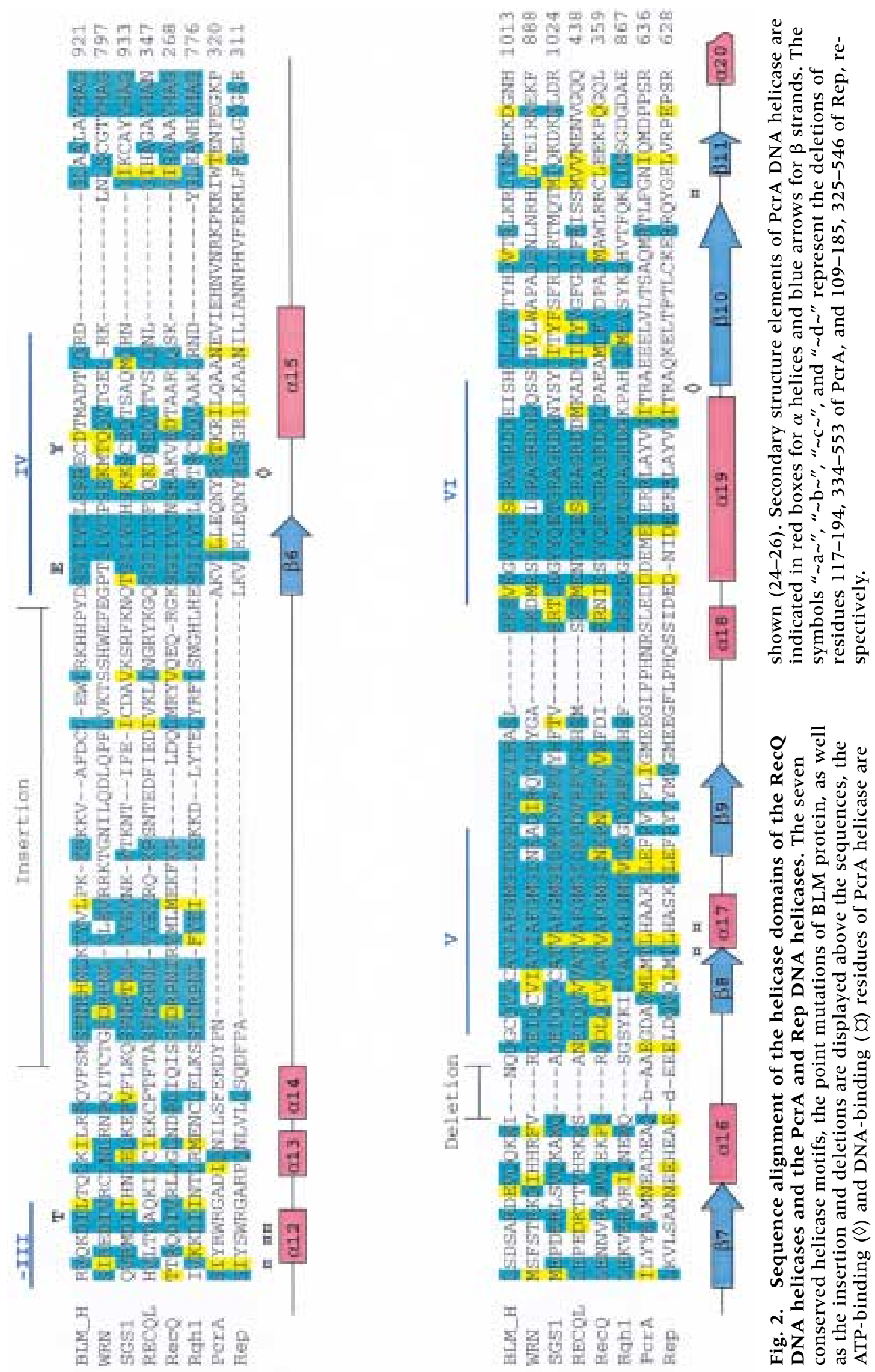
ture, helicase domain. Figure 3A shows the modeled BLM protein from residues 655 to 1029, superimposed with the template protein residues 4 to 652 . The PcrA helicase comprised four subdomains termed 1A, 1B, 2A and 2B; whereas, the BLM helicase domain model had only two subdomains, $1 \mathrm{~A}$ and $2 \mathrm{~A}$. In the model, the seven helicase motifs were situated between the subdomains $1 \mathrm{~A}$ and $2 \mathrm{~A}$. They formed the helicase core of the BLM protein (Fig. 3A,C-1), within which there were one cavity and another surface-exposed cleft that could be occupied by ATP and ssDNA, respectively (23-27). The final model showed good Ramanchandran plot and geometric parameters.

There are one insertion of 32 residues and two deletions of 77 and 215 residues in the model, in addition to other minor insertions and deletions (Fig. 2). The large insertion was located outside of subdomain $1 \mathrm{~A}$; whereas, the deletions of 77 and 215 residues were corre-

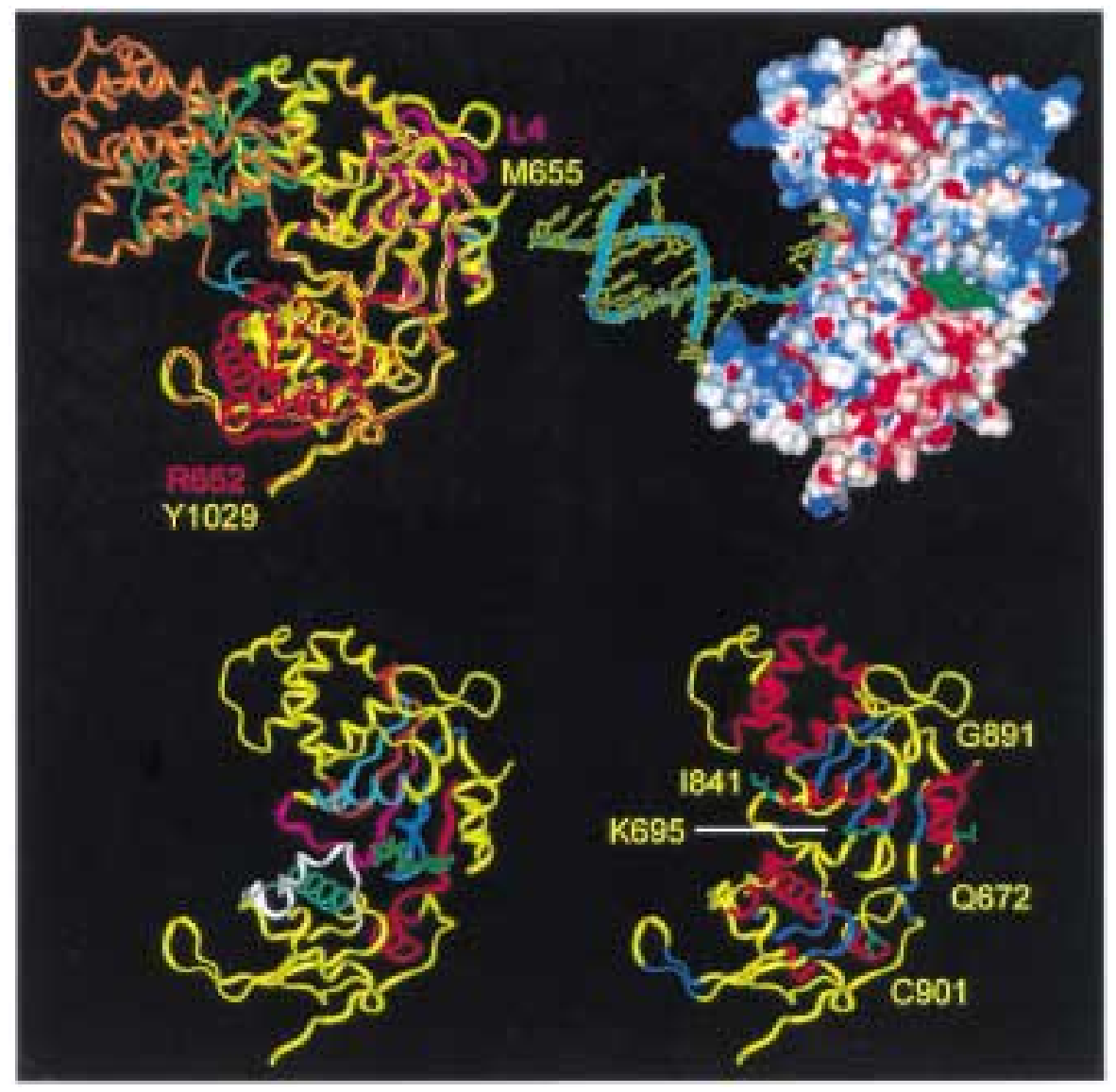

Fig. 3. Structural model of BLM helicase domain. (A) Superimposition of ribbon diagrams of the template protein PcrA DNA helicase (3PJR) and the BLM helicase domain (yellow). The trace of the PcrA helicase from residues 4 to 652 and the structural model of the BLM protein from residues 655 to 1029 are shown. The PcrA DNA helicase is comprised of four subdomains $1 \mathrm{~A}$ (magenta), $1 \mathrm{~B}$ (green), 2A (red) and 2B (orange); whereas, the BLM model only contains subdomains $1 \mathrm{~A}$ and $2 \mathrm{~A}$. (B) Electrostatic surface of the model with the same view as left. Negative potential is shown in red, positive potential in blue. The surface potential is negative over most of the surface except for the DNAbinding cleft. The docked ATP (green) is situated in the nucleotide-binding pocket between subdomains
1A and 2A. The merged DNA (yellow) with ribbon is located at the binding cleft with good complementarity. (C) Stereo diagram showing the structural model of the BLM helicase domain with the same view as above. (C-1) The seven conserved helicase motifs, I, Ia, II, III, IV, V and VI, are shown in blue, light blue, orange, magenta, red, white and green, respectively. The docked ATP is displayed in green. The nucleotide binding pocket is contributed by motifs I, II-IV and VI; whereas, the DNA binding cleft is formed by motifs Ia, III and V. (C-2) Secondary structure elements are indicated in red for $\alpha$-helices and blue for $\beta$-strands. The residues of BS-causing mutations are displayed with sidechains in green and residue K695, corresponding to mutation K703A on mouse BLM, is also displayed. 
sponding to the subdomain $\mathrm{IB}$ and the subdomain 2B, plus a small surface-exposed segment of subdomain $2 \mathrm{~A}$, respectively. Whether or not and how will these big insertion and deletions influence the model of the BLM helicase domain? This question is important to the application of the model and can be analyzed by further comparing the model to the structure of PcrA helicases.

Rep and PcrA helicases had four subdomains 1A, 2A, 1B and 2B (Fig. 3A). The ATPbinding pocket was located between subdomains $1 \mathrm{~A}$ and $2 \mathrm{~A}$ and consisted of helicase motifs I, II-IV and VI. The major component of the DNA-binding site, surface-exposed residues from motifs Ia, III and V, was situated at the surface cleft between subdomains $1 \mathrm{~A}$ and 2A; whereas, the subdomains $\mathrm{BB}$ and $2 \mathrm{~B}$ only formed the walls of the DNA-binding cleft and played a less important role (Figs. 3A, B). Obviously, the deletions of subdomains $1 B, 2 B$ and a small outside part of subdomain 2A did not influence the ATP binding. The deletion of subdomain $1 \mathrm{~B}$ had little influence on the DNA binding, because the major part of the binding site was composed of subdomains $1 \mathrm{~A}$ and $2 \mathrm{~A}$, while subdomain $\mathrm{IB}$ was only a minor part of the DNA binding site. The insertion, which could not be modeled, did not affect ATP and DNA binding because of its surface location.

The BLM protein has been shown to form oligomeric structure (43). Because of the low resolution of the electron microscopy structure, we were not able to localize the modeled part of the protein into the electron micrograph.

\section{Electrostatic Surface Potential}

The program Delphi (Molecular Simulations, Inc.) was applied to calculate the electrostatic potential for the model (Fig. 3B). The potential of the BLM helicase domain was highly negative over most of the surface with the exception of the cleft between subdomains $1 \mathrm{~A}$ and $2 \mathrm{~A}$, suggesting that the cleft could be involved in DNA binding. The surface potential of PcrA helicase was similar in the cleft (24). The bound DNA substrate from PcrA (26) was merged into the BLM helicase model and exhibited good complementarity. The model and the docked ATP also had good complementarity, with both electrostatic and spatial aspects.

\section{Discussion}

\section{Distribution of BLM Mutations}

The most common mutation in BS is delATCTGA/insTAGATTC at position 2281, known as blm ${ }^{A s h}$ mutation (@Y736X740). It is very common (carrier frequency 1/104) in the Ashkenazi Jewish population (44). BLMbase contained 10 patients from the Ashkenazi Jewish population. Almost all the Ashkenazi Jews with BS were homozygous for the $b_{l m}^{A s h}$ mutation (45). The mutation descriptions of 22 unique alleles are presented in BLMbase. The numbers of individual mutation types for different alleles were 6 missense mutations, 8 nonsense mutations, 3 frameshift insertions and 5 frameshift deletions. All the currently known BS-causing missense mutations are in the DEAH helicase or RecQCt domains. Seven nonsense or frameshift mutations led to stop codon and premature termination before the end of DEAH helicase domain. Two patients were heterozygous for BLM mutations. One patient had lbp insertion (1610insA), causing frameshift and stop codon in amino acid 516 in one allele (@G512X516) and a nonsense mutation in the other allele (Q752X). The other patient had a $b l m^{A s h}$ mutation in one allele and a lbp deletion (3233delT), causing frameshift and stop codon at the amino acid 1077. One frameshift deletion led to stop codon after RecQCt domain at the amino acid 1097. Lu et al. found that helicase domain may retain its DNA helicase activity when the C-terminus is truncated downstream of the helicase domain of SGS1, the BLM protein homolog in yeast $(19,46)$.

In addition to BLMbase, there is another database of BLM-causing mutations. Dr. James L. German maintains an other database called the Bloom Syndrome Registry $(35,47,48)$ that is not publicly available.

\section{Residues Involved in DNA Binding}

The structures of Rep and PcrA helicases free and bound to ssDNA (24-26) clearly showed that the motifs Ia, III and V contained amino acids contacting ssDNA (Fig. 2). Because the BLM helicase motifs sequentially and structurally match those of Rep and PcrA helicases, we infer that the DNA-binding site of BLM protein is located mainly in the motifs Ia, III and V. These motifs form the surface-exposed DNA-binding cleft between subdomains 1A and $2 \mathrm{~A}$ (Fig. 3B). In the model, the residues L716 and R717 in motif Ia; Q838, D840 and 
I841 in motif III; as well as T946 and A948 in motif $\mathrm{V}$ are corresponding to the DNA-binding residues in Rep and PcrA helicases (Fig. 2). Further evidence is provided that the mutation I841T in human BLM and the corresponding substitution I849T in mouse BLM $(3,4)$ impair the helicase and associated ATPase activities.

\section{Nucleotide Binding Site}

The ATP-binding residues (Fig. 2) of the Rep and PcrA helicases have been confirmed biochemically and structurally $(25,26)$. The corresponding residues in BLM protein, K695 and S696 in motif I, D795, and E796 in motif II, P835 in motif III, R899 in motif IV and S987 in motif VI, are thought to be ATP-binding residues. In the model, the motifs I, II-IV and VI are situated between subdomains $\mathrm{IA}$ and $2 \mathrm{~A}$ and form a pocket accommodated by nucleotide molecule (Fig. 3B,C-1). Based on the interaction mechanism of Rep and PcrA helicases with the nucleotide molecule (23-26), we further suggest that $\mathrm{K} 695$ and D795 are involved in coordinating $\mathrm{Mg}^{2+}$; whereas, S696 and E796 might play a catalytic role for ATP hydrolysis. Such a suggestion is supported by the observation that the point mutation K703A in mouse BLM, homologous to K695 in human BLM, abolished both ATPase and DNA-helicase activities (4). Moreover, the mutation $\mathrm{K} 196 \mathrm{H}$ in motif I of yeast SUGl, corresponding to K703 of mouse BLM and K695 of human BLM, had no detectable helicase or ATPase activity (49).

\section{Structural Basis of BS}

Four point mutations, Q672R, I841T, G891E and C901Y, have been identified in the human BLM helicase domain $(3,15)$ (Figs. 2 and 3C-2). These mutations are at the conserved positions and abolish both helicase and ATPase activities.

The mutation I841T, homologous to I849T of the mouse BLM protein, is situated at the end of motif III (Figs. 2 and 3C-1). I841 is surrounded by a cluster of charged residues, including R836, Q838, K839, D840 and Q844, which correspond to a cluster of ssDNAbinding residues Y248, W250 and R25 1 of Rep DNA helicase, forming hydrogen-binding interactions (23). The mutation I841T might affect the local electrostatics due to change from a hydrophobic residue to a hydrophilic amino acid. The substitution presumbly interferes with the hydrogen-binding interactions.
The mutations G891E and C901Y appear at the beginning and middle of motif IV, respectively (Figs. 2 and 3). Between G891 and C901, R899 corresponds to the ATP-contacting R287 of PcrA helicase $(25,26)$. The mutations G891E and C901Y could lead to electrostatic and spatial changes of ATP-binding pocket and probably disturb the local structure.

The mutation Q672R appears at the beginning of $\alpha$-helix 2 (Figs. 2 and 3C-2). The residue Q672, together with its neighboring negatively charged residues N671, E674 and N677, could form a part of the wall of the ATPbinding pocket. These residues correspond to a cluster of negatively charged residues, N13, E15, Q16, Q17 and E18, in the PcrA helicase $(25,26)$. The mutation leads to major electrostatic change at the position 672 , which presumably interferes with the orientation of ATP in the binding pocket and weakens its interactions with the BLM protein.

In addition, the mutation $\mathrm{K} 703 \mathrm{~A}$ on the mouse BLM protein is positionally homologous to K695 of the human BLM (Figs. 2 and 3C-2). The residue K703 corresponds to the conserved catalytic K37 of PcrA helicase, in which K37A mutation impaired helicase and ATPase activities $(25,26)$. The mutation K703A removes the charged sidechain, alters electrostatics and could eliminate the binding interaction. Furthermore, T38 in the PcrA helicase has been confirmed by mutation to play an important role during catalytic process (25). Thus, S704 in the mouse BLM or S696 in the human BLM should cause BS when mutated.

In summary, the mutations in the BLM helicase domain sharply decrease the helicase and ATPase activities, presumably by weakening interactions with DNA or ATP or by affecting the protein fold. In addition, we can further infer, based on other ATP-binding residues of PcrA helicase and their mutants (24-26), that there could be BS if D795 and E796 in motif II and N834 in motif III were mutated.

\section{Acknowledgments}

The financial support from the European Union (BioMed 2 PL 963007 and BioTech BIO4CT98-0142), Sigrid Juselius Foundation, the Medical Research Fund of Tampere University Hospital and National Technology Agency of Finland is gratefully acknowledged. 


\section{References}

1. German J. (1974) Bloom's syndrome. II. In: German J (ed.) Chromosomes and Cancer. John Wiley \& Sons, New Yok, pp. 601-617.

2. German J. (1993) Bloom syndrome: a Mendelian prototype of somatic mutational disease. Medicine 72: 393-406.

3. Ellis NA, Groden J, Ye TZ, et al. (1995) The Bloom's syndrome gene product is homologous to RecQ helicases. Cell 83: 655-666.

4. Bahr A, De Graeve F, Kedinger C, Chatton B. (1998) Point mutations causing Bloom's syndrome abolish ATPase and DNA helicase activities of the BLM protein. Oncogene 17: 2565-2571.

5. Morozov V, Mushegian AR, Koonin EV, Bork P. (1997) A putative nucleic acid-binding domain in Bloom's and Werner's syndrome helicases. Trends Biochem. Sci. 22: 417-418.

6. Kaneko H, Orii KO, Matsui E, et al. (1997) BLM (the causative gene of Bloom syndrome) protein translocation into the nucleus by a nuclear localization signal. Biochem. Biophys. Res. Commun. 240: 348-353.

7. Hanada K, Ukita T, Kohno Y, Saito K, Kato J, Ikeda H. (1997) RecQ DNA helicase is a suppressor of illegitimate recombination in Escherichia coli. Proc. Natl. Acad. Sci. U.S.A. 94: 3860-3865.

8. Gangloff S, McDonald JP, Bendixen C, Arthur L, Rothstein R. (1994) The yeast type I topoisomerase Top3 interacts with Sgs1, a DNA helicase homolog: a potential eukaryotic reverse gyrase. Mol. Cell Biol. 14: 8391-8398.

9. Watt PM, Louis EJ, Borts RH, Hickson ID. (1995) Sgsl: a eukaryotic homolog of E. coli RecQ that interacts with topoisomerase II in vivo and is required for faithful chromosome segregation. Cell 81: 253-260.

10. Stewart E, Chapman CR, Al-Khodairy F, Carr AM, Enoch T. (1997) rqh1+, a fission yeast gene related to the Bloom's and Werner's syndrome genes, is required for reversible $S$ phase arrest. EMBO J. 16: 2682-2692.

11. Seki M, Miyazawa H, Tada S, et al. (1994) Molecular cloning of cDNA encoding human DNA helicase Q1 which has homology to Escherichia coli Rec Q helicase and localization of the gene at chromosome 12p12. Nucleic Acids Res. 22: 4566-4573.

12. Puranam KL, Blackshear PJ. (1994) Cloning and characterization of RECQL, a potential human homologue of the Escherichia coli DNA helicase RecQ. J. Biol. Chem. 269: 29838-29845.

13. Yu CE, Oshima J, Fu YH, et al. (1996) Positional cloning of the Werner's syndrome gene. Science 272: 258-262.

14. Lohman TM, Bjornson KP. (1996) Mechanisms of helicase-catalyzed DNA unwinding. Annu. Rev. Biochem. 65: 169-2 14.
15. Ellis NA. (1997) DNA helicases in inherited human disorders. Curr. Opin. Genet. Dev. 7: 354-363.

16. Gorbalenya AE, Koonin EV, Donchenko AP, Blinov VM. (1989) Two related superfamilies of putative helicases involved in replication, recombination, repair and expression of DNA and RNA genomes. Nucleic Acids Res. 17: 4713-4730.

17. Karow JK, Chakraverty RK, Hickson ID. (1997) The Bloom's syndrome gene product is a $3^{\prime}-5^{\prime}$ DNA helicase. J. Biol. Chem. 272: 30611-30614.

18. Gray MD, Shen JC, Kamath-Loeb AS, et al. (1997) The Werner syndrome protein is a DNA helicase. Nat. Genet. 17: 100-103.

19. Lu J, Mullen JR, Brill SJ, Kleff S, Romeo AM, Sternglanz R. (1996) Human homologues of yeast helicase. Nature 383: 678-679.

20. Bennett RJ, Sharp JA, Wang JC. (1998) Purification and characterization of the Sgs l DNA helicase activity of Saccharomyces cerevisiae. J. Biol. Chem. 273: 9644-9650.

21. Foucault F, Vaury C, Barakat A, et al. (1997) Characterization of a new BLM mutation associated with a topoisomerase II alpha defect in a patient with Bloom's syndrome. Hum. Mol. Genet. 6: 1427-1434.

22. Ellis NA, Ciocci S, Ye T, Neff N, German J. (1997) Mutational analysis of the BLM gene in nonAshkenazi-Jewish persons with Bloom's syndrome. Am. J. Hum. Genet. 61 supplement: A332.

23. Korolev S, Hsieh J, Gauss GH, Lohman TM, Waksman G. (1997) Major domain swiveling revealed by the crystal structures of complexes of E. coli Rep helicase bound to single-stranded DNA and ADP. Cell 90: 635-647.

24. Subramanya HS, Bird LE, Brannigan JA, Wigley DB. (1996) Crystal structure of a DExx box DNA helicase. Nature 384: 379-383.

25. Soultanas P, Dillingham MS, Velankar SS, Wigley DB. (1999) DNA binding mediates conformational changes and metal ion coordination in the active site of PcrA helicase. J. Mol. Biol. 290: 137-148.

26. Velankar SS, Soultanas P, Dillingham MS, Subramanya HS, Wigley DB. (1999) Crystal structures of complexes of PcrA DNA helicase with a DNA substrate indicate an inchworm mechanism. Cell 97: 75-84.

27. Gorbalenya AE, Koonin EV. (1993) Helicase: amino acid sequence comparisons and structure-function relationships. Curr. Opin. Struct. Biol. 3: 419-429.

28. Bird LE, Brannigan JA, Subramanya HS, Wigley DB. (1998) Characterisation of Bacillus stearothermophilus PcrA helicase: evidence against an active rolling mechanism. Nucleic Acids Res. 26: 2686-2693.

29. Vihinen M, Kwan SP, Lester T, et al. (1999) Mutations of the human BTK gene coding for bruton tyrosine kinase in X-linked agammaglobulinemia. Hum. Mutat. 13: 280-285. 
30. Etzold T, Argos P. (1993) Transforming a set of biological flat file libraries to a fast access network. Comput. Appl. Biosci. 9: 59-64.

31. Thompson JD, Higgins DG, Gibson TJ. (1994) CLUSTAL W: improving the sensitivity of progressive multiple sequence alignment through sequence weighting, position-specific gap penalties and weight matrix choice. Nucleic Acids Res. 22: 4673-4680.

32. Hobohm U, Sander C. (1994) Enlarged representative set of protein structures. Protein Sci. 3: 522-524.

33. Hobohm U, Scharf M, Schneider R, Sander C. (1992) Selection of representative protein data sets. Protein Sci. 1: 409-417.

34. Rodriguez R, Chinea G, Lopez N, Pons T, Vriend G. (1998) Homology modeling, model and software evaluation: three related resources. Bioinformatics 14: 523-528.

35. German J. (1997) Bloom's syndrome. XX. The first 100 cancers. Cancer Genet. Cytogenet. 93: 100-106.

36. Kaneko H, Inoue R, Yamada Y, et al. (1996) Microsatellite instability in B-cell lymphoma originating from Bloom syndrome. Int. J. Cancer. 69: 480-483.

37. Calin G, Herlea V, Barbanti-Brodano G, Negrini M. (1998) The coding region of the bloom syndrome BLM gene and of the CBL proto-oncogene is mutated in genetically unstable sporadic gastrointestinal tumors. Cancer Res. 58: 37773781.

38. The Coriell Institute for Medical Research. (1999) Characterized mutations in the BLM gene. In: The National Institute of General Medical Sciences (NIGMS) Human Genetic Mutant Cell Repository. http://locus.umdnj.edu/nigms/ charmut/bloom.html, Repository numbers: GM00811，GM01492，GM02085，GM03402, GM03498, GM03510, GM09960. Camden, New Jersey. Accessed on September 15, 1999.

39. Riikonen P, Vihinen M. (1999) MUTbase program package for maintenance and analysis of mutation databases on the World Wide Web. Bioinformatics 15: 852-859.
40. Cuff JA, Clamp ME, Siddiqui AS, Finlay M, Barton GJ. (1998) JPred: a consensus secondary structure prediction server. Bioinformatics 14: 892-893.

41. Cuff JA, Barton GJ. (1999) Evaluation and improvement of multiple sequence methods for protein secondary structure prediction. Proteins 34: 508-519.

42. Laskowski RA, Hutchinson EG, Michie AD, Wallace AC, Jones ML, Thornton JM. (1997) PDBsum: a Web-based database of summaries and analyses of all PDB structures. Trends Biochem. Sci. 22: 488-490.

43. Karow JK, Newman RH, Freemont PS, Hickson ID. (1999) Oligomeric ring structure of the Bloom's syndrome helicase. Curr. Biol. 9: 597-600.

44. Roa BB, Savino CV, Richards CS. (1999) Ashkenazi Jewish population frequency of the Bloom syndrome gene 2281 delta 6ins7 mutation. Genet. Test. 3: 219-221.

45. Li L, Eng C, Desnick RJ, German J, Ellis NA. (1998) Carrier frequency of the Bloom syndrome $b_{l m}^{A s h}$ mutation in the Ashkenazi Jewish population. Mol. Genet. Metab. 64: 286-290.

46. Neff NF, Ellis NA, Ye TZ, et al. (1999) The DNA helicase activity of BLM is necessary for the correction of the genomic instability of bloom syndrome cells. Mol. Biol. Cell 10: 665-676.

47. German J, Ellis NA, Proytcheva M. (1996) Bloom's syndrome. XIX. Cytogenetic and population evidence for genetic heterogeneity. Clin. Genet. 49: 223-231.

48. German J, Passarge E. (1989) Bloom's syndrome. XII. Report from the Registry for 1987. Clin. Genet. 35: 57-69.

49. Fraser RA, Rossignol M, Heard DJ, Egly JM, Chambon P. (1997) SUGl, a putative transcriptional mediator and subunit of the PA700 proteasome regulatory complex, is a DNA helicase. J. Biol. Chem. 272: 7122-7126.

50. Vihinen M, Leväslaiho H, Cotton RD. (1999) Immunodeficiency mutation databases. In: Ochs HD (ed.) Primary Immunodeficiency Diseases. A Molecular and Genetic Approach. Oxford University Press, New York, Oxford, pp. 443-447. 